\title{
Antipodal Detection and Decoding for Large Multi-User MIMO with Reduced Base-Station Antennas
}

\author{
Christopher Husmann, Rahim Tafazolli and Konstantinos Nikitopoulos \\ 5G Innovation Centre \\ Institute for Communication Systems \\ University of Surrey, Guildford, UK \\ Email: \{c.husmann, r.tafazolli, k.nikitopoulos\}@ surrey.ac.uk
}

\begin{abstract}
To avoid unnecessarily using a massive number of base station antennas to support a large number of users spatially multiplexed multi-user MIMO systems, optimal detection methods are required to demultiplex the mutually interfering information streams. Sphere decoding (SD) can achieve this, but its complexity and latency becomes impractical for large MIMO systems. Low complexity detection solutions such as linear detectors (e.g., MMSE) or likelihood ascendant search (LAS) approaches, have significantly lower latency requirements than SD but their achievable throughput is far from optimal. This work presents the concept of Antipodal detection and decoding, that can deliver very high throughput with practical latency requirements, even in systems where the number of user antennas reaches the number of base station antennas. The Antipodal detector either results in a highly reliable vector solution, or it does not find a vector solution at all (i.e., it results in an erasure), skipping the heavy processing load related to finding vector solutions that have a very high likelihood to be erroneous. Then, a belief-propagation-based decoder is proposed, that restores these erasures and further corrects remaining erroneous vector solutions. We show that for $32 \times 32$, 64-QAM modulated systems, and for packet error rates below $10 \%$, Antipodal detection and decoding requires $9 \mathrm{~dB}$ less transmitted power than systems employing soft MMSE or LAS detection and LDPC decoding with similar complexity requirements. For the same scenario, our Antipodal method achieves practical throughput gains of more than 50\% compared to soft MMSE and soft LAS-based methods.

Index Terms-MIMO systems, belief-propagation decoding, spatial multiplexing
\end{abstract}

\section{INTRODUCTION}

One of the most important challenges in the design of next generation wireless communication systems is to meet users' ever-increasing demand for capacity and throughput [1]. Large Mulitple-Input-Mulitple-Output (MIMO) systems with spatial multiplexing are among the most promising ways to satisfy this demand and, therefore, it will be a key technology in upcoming cellular [2] and local-area [3] networks.

Although, spatial multiplexing can increase the net throughput in both the uplink and downlink directions of wireless communication systems, here we focus on the uplink case where a large number of users concurrently transmit information to a multi - antenna base station.
To take full advantage of the potential of spatial multiplexing, and to avoid the current trend of unnecessarily using a massive number of base - station antennas to support a large but limited number of users there is a challenging prerequisite. We need to be able to optimally demultiplex the mutually interfering information streams. Sphere decoding (SD) is a wellknown technique that dramatically reduces the complexity for optimally, in the maximum-likelihood (ML) sense, detecting mutually interfering information streams by translating the ML detection problem into a tree search problem [4], [5]. ML detection is known from theory to minimize detection errors and, therefore, it can substantially outperform simple, but suboptimal, linear detection schemes like zero-forcing (ZF) and mimimum-mean-square-error (MMSE). While the throughput gains of sphere decoding increase with the number of mutually interfering information streams, the corresponding processing complexity becomes impractical for high-order modulation alphabets and for large numbers of mutually interfering information streams [6], [7]. The recently proposed Geosphere [8], [9] algorithm enables the transmission of very dense symbol constellations (e.g., 1024-QAM), however with a complexity that still increases exponentially with the number of the mutually interfering information streams. In addition, traditional sphere decoding provides "hard" information (i.e., detected symbols) instead of "soft information" (likelihoods) that is required in order to perform a-posteriori probability (APP) decoding and apply powerful state-of-the-art coding schemes (e.g., LDPC coding). Extensions that deliver "soft information" are indeed known [10], [11], but their complexity and latency requirements are beyond the capabilities of current processors for targeting very large MIMO systems.

Examples of low-complexity non-linear MIMO detectors exist in the family of "local neighborhood search", including the complexity efficient likelihood ascendant search (LAS) algorithm [12] and the reactive tabu search (RTS) algorithm [13]. By levering the asymptotic characteristics of MIMO systems with massive numbers of mutually interfering information, these approaches are able to approach ML performance at a fraction of the SD complexity. Their performance however degrades drastically when transmitting dense sym- 
bol constellations or for moderate numbers (of the order of tens) of interfering streams. Furthermore, similarly to sphere decoding, traditional local neighborhood search algorithms provide only "hard" information. To enable powerful APP decoding schemes like LDPC, LAS has been extended to provide approximate soft-information [14] but again such an approximation is only efficient for low-order symbol constellations (e.g., 4-QAM).

In this work we present the concept of Antipodal Detection and Decoding, that has been designed to deliver very high throughput in large MIMO systems with a practical number (in the order of tens) of interfering streams. In contrast to local neighbourhood searches, the Antipodal detector can efficiently utilize very dense constellations (e.g., 256-QAM) and it "naturally" (at no extra costs) supports powerful LDPC channel coding. In addition, Antipodal processing can adjust its complexity and latency in order to meet the limitations imposed by the base station's hardware and the systems needs.

Antipodal detection and decoding is based on a simple observation. In order for a vector solution to be highly reliable, there should be no other (or only a few) candidate vector with similar Euclidean distance to the received signal. When this holds, proposed SD tree pruning approaches can drastically reduce the search space and consequently the ML solution can be identified fast. On the other hand, if a solution is less reliable, this practically means that there are many candidate solutions with a similar Euclidean distance to the received signal. Then the SD has to visit a significantly higher number of possible solution before identifying the ML one, resulting in substantially increased complexity and latency. In other words, most of the SD's processing complexity and latency is devoted to find unreliable solutions. Therefore, instead of wasting the vast majority of the resources to identify vector solutions that have a significant likelihood to be erroneous, we focus the available processing power on identifing reliable solutions and leave the identification of the rest to the Antipodal decoder.

In particular, the Antipodal detector is realized by means of a "depth-first" sphere decoder with statistical pruning. The Antipodal pruning approach improves on [15] and leverages the noise statistics to define strict pruning conditions which promptly exclude vectors that have a small likelihood to be the correct solution. When the Antipodal detector finds, within strict latency limitations, a vector that passes all pruning checks this vector is classified as highly reliable. Otherwise, the transmitted vector is treated as an erasure. Consequently, the outcome will be Antipodal. The detector result is either highly reliable or is an erasure (e.g., no solution has been found). To handle the erasure traditional LDPC decoders could be employed. However, we propose a specifically tailored Antipodal decoder that is based on the belief-propagation principle. Exploiting the Antipodal decoder input, we can significantly simplify the internal operation of the decoder. Additionally, the proposed decoder introduces an iterative technique that can identify detection errors in the as highly reliable classified solutions. As a result, the Antipodal decoder can reduce the achievable packet error rate (PER) by an order of magnitude compared with traditional LDPC decoders. In extensive simulations, we show that Antipodal detection and decoding with practical complexity requirements significantly outperforms linear and non-linear decoding strategies by more than $10 \mathrm{~dB}$.

\section{System Model}

A $N_{t} \times N_{r}$ flat-fading multi-user MIMO channel is assumed where multiple single-antenna users concurrently transmit LDPC encoded streams to a multi-antenna access point. When transmitting the vector $\mathbf{s}$ over a flat-fading communication channel with $N_{t}$ mutually interfering transmission stream to $N_{r}$ receiving entities (e.g antennas), the received vector is given by

$$
\mathbf{y}=\mathbf{H s}+\mathbf{n},
$$

with $\mathbf{H}$ being the $N_{r} \times N_{t}$ MIMO channel matrix. The $N_{t}$ elements of the transmit vector $\mathbf{s}$ belong to a complex constellation $\mathcal{Q}$ and the set of possible transmission vectors is denoted as $|\mathcal{Q}|^{N_{t}}$. The $N_{r}$ dimensional vector $\mathbf{n}$ denotes the additive white Gaussian noise (AWGN).

\section{SPHERE DECODER}

To put the Antipodal Detector into perspective, this Section revists the principles of "depth-first" sphere decoding with the Schorr-Euchner enumeration and radius update, which is a particularly efficient method for solving the ML problem. Within the employed system model the ML problem is defined as

$$
\mathbf{s}_{\mathrm{ML}}=\arg \min _{s \in|\mathcal{Q}|^{N_{t}}}=\|\mathbf{y}-\mathbf{H s}\|^{2} .
$$

SD simplifies the minimization problem by transforming the problem into an equivalent tree search [8]. In particular, by QR decomposing the MIMO channel matrix as $\mathbf{H}=\mathbf{Q R}$, where $\mathbf{Q}$ is a orthonormal matrix and $\mathbf{R}$ is an upper triangular matrix, the ML problem can be transformed into:

$$
\mathbf{S}_{\mathrm{ML}}=\arg \min _{s \in|\mathcal{Q}|^{N_{t}}}\|\overline{\mathbf{y}}-\mathbf{R s}\|^{2} \text {. }
$$

with $\overline{\mathbf{y}}=\mathbf{Q}^{*} \mathbf{y}$. The tree has a height of $N_{t}$ and a branch factor of $|\mathcal{Q}|$. Each level $l$ of the tree is related to the symbol transmitted from a specific antenna. In addition, each node of a specific level $l$ is associated with a partial symbol vector $\mathbf{s}_{l}=\left[s\left(N_{t}-l\right), . ., s\left(N_{t}\right)\right]$ containing all potential transmitted symbols down to this level, and it is characterized by its Partial euclidean Distance (PD) [4]

$$
c\left(\mathbf{s}_{l}\right)=\left(\bar{y}(l)-\sum_{p=l}^{N_{t}} R(l, p) \cdot s_{l}(p)\right)^{2}+c\left(\mathbf{s}_{l+1}\right),
$$

with $R(k, p)$ being the element of $\mathbf{R}$ at the $k^{\text {th }}$ column and the $p^{\text {th }}$ row and $\bar{y}(l)$ being the $1^{\text {th }}$ element of the vector $\overline{\mathbf{y}}$. The PD of the root of the tree $c\left(s_{N_{t}+1}\right)$ is zero. Then, the ML problem is translated into finding the leaf node with the minimum $c\left(\mathbf{s}_{1}\right)$. For depth-first sphere decoders with SchnorrEuchner enumeration and radius reduction [16] the radius is initially set to infinity. Then, whenever a leaf $\mathbf{s}_{1}$ is reached with 
its PD less than the squared radius $r^{2}$, the radius is updated to $c\left(\mathbf{s}_{1}\right)$. Upon meeting a node $\mathbf{s}_{l}$, if $c\left(\mathbf{s}_{l}\right)>r^{2}$ this node, its children and this nodes siblings that have not been visited are all pruned. To define the search order, according to the Schnorr-Euchner enumeration, the children of a parent node are visited in ascending order of their PDs [16].

\section{Antipodal Detection And Decoding}

The proposed method consists of the Antipodal detector and the Antipodal decoder. (i) The Antipodal detector is realized by meas of a "depth-first" SD approach in combination with statistical pruning. The proposed pruning in combination with the tight complexity limitations promptly exclude unreliable solutions from the search space. As a consequence all solutions found are reliable. If no reliable solution exists then the transmitted vector is treated as an erasure. (ii) The Antipodal decoder is realized through a tailored to the Antipodal input belief-propagation approach. The Antipodal decoder first restores the bits that have been erased by the Antipodal detector. Then, an iterative method is utilized to corrected the erroneously detected bits. To maximize the synergy between Antipodal detection and Antipodal decoding, the employed statistical pruning is adjusted according to the correction capability of the applied LDPC code.

\section{A. Antipodal Detector}

The antipodal Detector employs a pruning approach, according to which a vector solution, or a set of vector solutions, is excluded from the search if one or several pruning conditions are met. As a result, the pruning approach reduces the search space. The probabilistic pruning metric is based on the statistics of the AWGN. In particular, a pruning condition is defined for each layer of the search tree. For the $l$-th layer, the pruning metric is

$$
r_{\mathrm{p}}(l)=\sigma^{2} \cdot F_{\chi^{2}\left(2\left(N_{t}-l+1\right)\right)}^{-1}(1-\beta) .
$$

Where $r_{\mathrm{p}}(l)$ is the pruning metric of layer $l$ of the search tree, $F_{\chi^{2}(N)}^{-1}$ is the inverse of the cumulative distribution function (CDF) of the chi-squared distribution with $N$ degrees of freedom, and $\beta$ is the pruning parameter. During the tree traversal, if the PD metric of a node is larger that the pruning metric of the level in which the particular node is located, this node, its siblings and all its children nodes are pruned. A vector solution is characterized as being highly reliable when only one vector solution passes all pruning conditions. To quantify the reliability of an accepted solution we define $\mathbf{s}^{\star}$ as the transmitted symbol vector. Then, all symbol vectors $\tilde{\mathbf{S}}$ that fulfill the following properties:

$$
\begin{aligned}
\tilde{\mathbf{s}}(p)-\mathbf{s}^{\star}(p) & =0, \quad \forall p \neq l \\
\tilde{\mathbf{s}}(l)-\mathbf{s}^{\star}(l) & =\left|D_{\min }\right|,
\end{aligned}
$$

(where $D_{\min }$ is the minimum distance between two symbols of the utilized constellation) build the set $\Theta$. Since the vectors in $\Theta$ have the smallest Euclidean distance to the transmitted symbol vector, they are the most likely vectors to cause a detection error. Therefore, we approximate the reliability of an accepted vector solution, by the probability that none of the vectors in $\Theta$ pass the pruning check in the last layer of the SD tree:

$$
\begin{aligned}
P\left(\hat{\mathbf{s}} \neq \mathbf{s}^{\star}\right) & <P\left(c\left(\hat{\mathbf{s}}_{1}\right)<r_{\mathrm{p}}(1)\right) \\
& \approx P\left(c\left(\tilde{\mathbf{s}}_{1}\right)<r_{\mathrm{p}}(1) \mid \forall \tilde{\mathbf{s}} \in \Theta\right) .
\end{aligned}
$$

Using (6) and (7), the $c\left(\tilde{\mathbf{s}}_{1}\right)$ is calculated as:

$$
\begin{aligned}
c\left(\tilde{\mathbf{s}}_{1}\right) & =\|\mathbf{y}-\mathbf{H} \tilde{\mathbf{s}}\|^{2} \\
& =\left\|\mathbf{H}\left(\mathbf{s}^{\star}-\tilde{\mathbf{s}}\right)+\mathbf{n}\right\|^{2},
\end{aligned}
$$

that can be expressed as:

$$
c\left(\tilde{\mathbf{s}}_{1}\right)=\sum_{p=1}^{N_{t}}\left\|H(p, l) \cdot D_{\min }+n(l)\right\|^{2} .
$$

For the employed system model, $H(p, l)$ and $n(l)$ are i.i.d. Gaussian distributed random variable. Due to that, the random variable $D_{\min } \cdot H(p, l)+n(l)$ is also Gaussian distributed

$$
\left(H(p, l) \cdot D_{\min }+n(l)\right) \sim \mathcal{N}\left(0, D_{\min }^{2}+\sigma^{2}\right) .
$$

From (11) follows that $c\left(\tilde{\mathbf{s}}_{1}\right)$ is a $\chi^{2}$ distributed random variable. Then, (8) can be written as

$$
\begin{aligned}
P\left(\hat{\mathbf{s}} \neq \mathbf{s}^{\star}\right) & <P\left(c\left(\hat{\mathbf{s}}_{1}\right)<r_{\mathrm{p}}(1)\right) \\
& \approx 4 \cdot N_{t} \cdot F_{\chi^{2}\left(2 N_{t}\right)}\left(\frac{\sigma^{2} \cdot r_{\mathrm{p}}(1)}{D_{\min }^{2}+\sigma^{2}}\right) .
\end{aligned}
$$

The pruning boundary $r_{\mathrm{p}}(1)$ (defined in (5)) is monotonically decreasing function of the pruning parameter $\beta$. Decreasing the value of the pruning parameter increases the reliability of the outcome of the Antipodal Decoder. Here we employ the statistics of the Rayleigh fading channel to analytically show the relationship between the pruning parameter and the likelihood of the resulting vector solution to be erroneous. Still, the same pruning approach can be used in any channel, independently of its statistics. To maximize the synergy between the Antipodal Detection and Decoding the parameter $\beta$ is adjusted in a way that the probability $\left(P_{E r}\right)$ of not finding a solution is below a threshold $\tau_{E}$, where $\tau_{E}$ is chosen with respect to the erasure tolerance of the applied decoder.

\section{B. Antipodal Decoder}

In this work, the Antipodal decoder is a modification of a traditional belief-propagation-decoder tailored to the Antipodal detector output. Exploiting the the Antipodal nature of the detector output, we could significantly simplify the internal operation of the proposed decoder. Specifically, the Antipodal decoder employs only binary additions to correct the detector's output. We note that, while some detected bits are characterized as being highly reliable, bit errors may still occur in practice. Therefore, the Antipodal Decoder utilizes an iterative technique to correct the erroneous bits that have been falsely classified as being reliable. As a result and in particular for long codewords lengths, the proposed decoding structure has a superior decoding performance compared to traditional beliefpropagation decoders. 


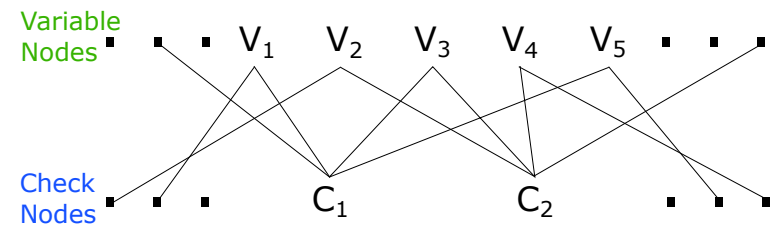

Fig. 1. Partial Decoding Graph

Let vector $\mathbf{d}$ contain the Antipodal outcome of the detected bits for a transmitted packet. The vector's dimension is $1 \times B$ with $B$ being the packet length. Its elements take one of the three values $[0,1, e]$, where $e$ represents an erased bit (i.e., no outcome) and $[0,1]$ represents highly reliable bits as have been detected from the Antipodal detector (after symbol-tobit-mapping).

Erasure Recovery: Similar to a traditional beliefpropagation decoder, the Antipodal Decoder can be described in terms of a factor graph. The nodes within the graph are separated into variable nodes and check nodes, where the variable nodes are only connected to check nodes and the check nodes are only connected to variable nodes. A variable node $V_{i}$ corresponds to the $i^{\text {th }}$ coded bit in the encoded packet, and each check node represents one parity check equation as determined by the employed LDPC code, as shown in Figure 1. To recover the erased bits, the Antipodal decoder creates a list $\mathcal{L}_{e}$ of all check nodes connected to at least one variable node representing an erased bit. Then, the detector tests if the first node on the list $\mathcal{L}_{e}$ is "resolvable". Namely, if the value of the erased bit can be calculated using the parity check equation at the specific check node

$$
V_{e}=\sum_{\forall i \in \Phi, i \neq e} V_{i}
$$

where $\Phi$ is the set of indexes of the variable nodes connected to the resolvable check node, and $V_{e}$ is the variable node where the related erasure lies. When the decoder visits a resolvable check node, it recovers the corresponding erased bit and deletes the check node from the $\mathcal{L}_{e}$ before continuing to the next node in the list. If the visited check node is not resolvable the decoder moves to the next check node in $\mathcal{L}_{e}$. Several iterations of this process may take place before decoding is terminated. In particular, the Antipodal decoder revisits the list $\mathcal{L}_{e}$ either until it becomes empty, or when no additional erasures have been recovered during an iteration. Even if the detector has characterized a detected outcome, and therefore the corresponding bits, as being highly reliable, in practice some of these bits may still be erroneous. The proposed decoder has the ability to identify such cases, and take further steps to improve the final detection/decoding performance. Specifically, when all erasures have been recovered and the parity checks across all check nodes are correct, the decoder output can be characterized as being highly-reliable. If not, the decoder output is characterized as non-reliable and it is an indication that a detection error may be present. Therefore, the decoding outcome is also Antipodal.
Bit Correction: As discussed before, the bits detected by the Antipodal detector may have been classified as highly reliable, but still in practice detection errors occasionally happen. One erroneously detected bit can corrupt the whole codeword and results in an undecodable packet. Assuming uncorrelated detection errors, and a bit-error-rate of $\tau$ for all bits, the probability that a packet error is caused by wrongly detected bits, follows a geometric distribution

$$
\begin{aligned}
P_{\tau} & =\sum_{l=1}^{L}\left(\left(\begin{array}{l}
l \\
B
\end{array}\right) \cdot(\tau)^{l} \cdot(1-\tau)^{L-l}\right), \\
& \approx B \cdot \tau .
\end{aligned}
$$

The approximation (14) holds for tau that are small relative to the packet length $B\left(\tau \ll B^{-1}\right)$. Further, the approximation shows that most of the packet errors induced by erroneously detected bits, are caused by only one flipped bit. Based on the above rationale, our proposed approach operates under the hypothesis that a packet error has been caused by a single bit error.

The Antipodal decoder distinguishes between exposed and hidden bit errors. To clarify the difference between the two different error types we introduce the notation of "erasure free" check nodes. A check node is "erasure free" if it is not connected to any variable node that is associated with an erased bit. Then, a bit error is exposed if the corresponding variable node is connected to at least one "erasure free" check node.

An exposed bit error can be easily recognized before the first decoding attempt. In particular, if the parity check of any "erasure free" check node is violated, at least one of the connected variable nodes is associated with the erroneous bit. In such cases, the proposed decoder simply intentionally erases all bits connected to this check node and later recovers them together with the Antipodal detector erasures.

A hidden bit error is more difficult to locate since the corresponding variable node is not connected to any erasure free check node. The Antipodal decoder requires multiple iterations to locate and corrected such a hidden error. After the first attempted to recover the erasures, a hidden error will cause several parity check violations. In the best case, only the check nodes directly connected to the variable node associated with the erroneously detected bit would fail the parity checks. Unfortunately, some erased bits might been recovered based on the erroneous bits and consequently the resolved bits are also erroneous (see (13)). Thus, it is possible that the variable node associated with the initial erroneous detected bit is not connected to any of the check nodes with unsuccessful parity checks. This makes the correction of the hidden bit error challenging. Initially the location of a hidden error can only be narrowed down to bits that have been classified as reliable. Thus, the variable nodes corresponding to those bits are saved in the list of possible error locations $\mathcal{L}_{L}$. After an unsuccessful erasure recovery, the decoder, for each of the variable's node in $\mathcal{L}_{L}$, sums its connections to check nodes with unsuccessfully parity check. 
Then, all the variable nodes sharing the maximum number of such connections constitute the list of "doubtful" nodes. After temporary saving the bits associated to these doubtful nodes the decoder changes the value of the corresponding elements in $\mathbf{d}$ from 1 or 0 to erasure, and restarts the erasure recovery process. If the erasure recovery is successful it means that the hidden bit error was associated with one doubtful variable node. Otherwise, the hidden bit error was not associated to one doubtful variable node. Therefore, the decoder restores the corresponding original bit values (i.e., the 1 or 0 that has been temporarily erased) in $\mathbf{d}$ and deletes the doudtful variable nodes from the list of possible error locations $\mathcal{L}_{L}$. Before the next erasure recovery attempt, the decoder rebuilds the set of of suspicious nodes, based on the updated list $\mathcal{L}_{L}$. The decoding algorithm terminates when a corrected codeword is found or when the maximum number of iterations is reached. At each decoding iteration the decoder reshuffles the list $L_{e}$ in order to increase the likelihood to find the hidden bit error.

\section{Evaluation}

In this Section we evaluate the throughput performance of the Antipodal detection and decoding method through extensive simulations. The proposed method is evaluated under practical complexity/latency requirements and is compared to other soft (LDPC encoded) and hard (convolutionally encoded) detection/decoding schemes of similar complexity. The MIMO channel is modeled as Rayleigh fading and it is assumed static per transmitted packet. A coding rate of 0.75 is adopted and 4, 16, 64 and 256-QAM constellations are considered. The Antipodal method is compared against three benchmark detection/decoding schemes of similar complexity. The first scheme is LDPC encoded, with the generator matrix of IEEE 802.11n [17], and it employs a soft MMSE detector and a belief-propagation channel decoder. The second scheme is again LDPC encoded, and a LAS detector that provides softinformation is utilized [14]. The third scheme is convolutionally encoded [18] and it uses the hard version of the LAS detection algorithm [12] followed by a Viterbi decoder [18]. Comparisons with optimal ML decoders (e.g. sphere decoding) have not been included due to their corresponding prohibitive complexity. For example, in a $32 \times 32$, 64-QAM modulated system, at an SNR of $28 \mathrm{~dB}$ a traditional sphere decoder [4] requires an average of $10^{8}$ complex multiplications to process just a single received symbol vector. Since the proposed detector is an SD-based approach, a one-visitednode-per-cycle architecture, can be assumed [9]. This allows us to model any practical latency constraints by means of $\alpha N_{t}$ visited nodes (or clock cycles). For the rest of this Section, unless explicitly stated otherwise, an $\alpha=16$ and a pruning parameter $\beta=0.025$ is assumed.

Fig. 2 shows the achievable throughput of Antipodal detection and decoding in comparison with the corresponding throughput achieved by the three other detection/decoding schemes. While the throughput performance of the three evaluated schemes is comparable in the case of 4-QAM, the proposed methods outperforms the MMSE based detection and

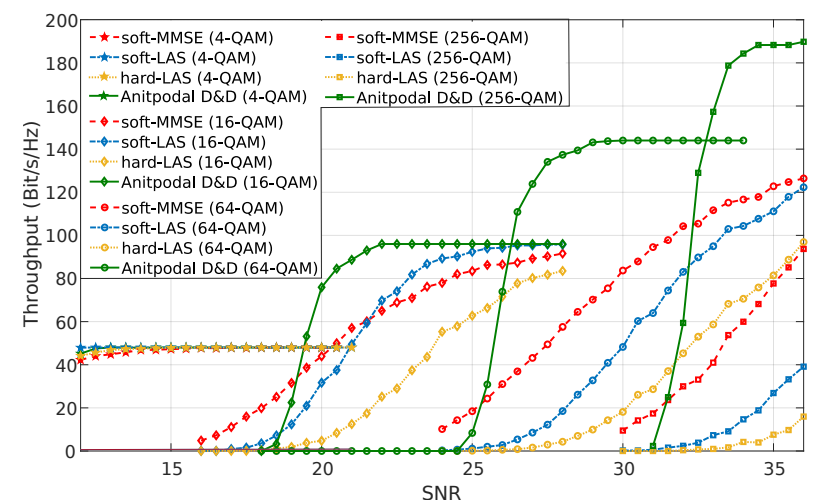

Fig. 2. Throughput of Antipodal detection and decoding compared with other detection and decoding scheme of similar complexity in a $32 \times 32$ MIMO system using code rate of 0.75 .

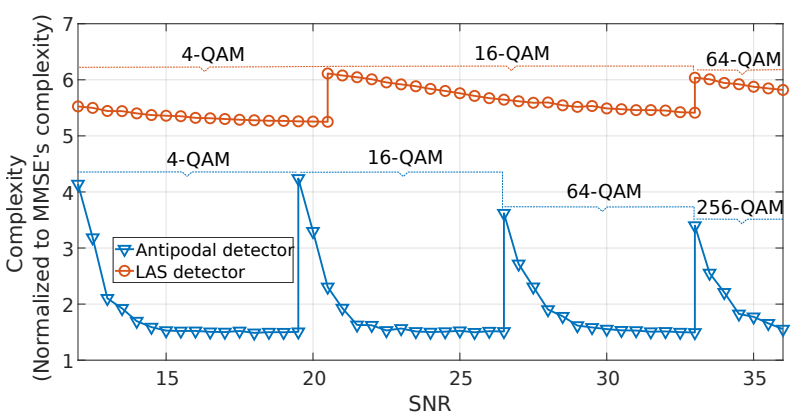

Fig. 3. Detection complexity of Antipodal detector (triangle) and LAS detector (circle) normalized to MMSE's detection complexity, in a $32 \times 32$ MIMO system using code rate of 0.75 .

decoding scheme by $5.5 \mathrm{~dB}$ when 16-QAM is used and by $9 \mathrm{~dB}$ when 64-QAM symbols are transmitted. Notice also that the proposed method significantly outperforms the non-linear LAS detection regardless of the utilized decoding strategy. For example, using 64-QAM modulation and LAS in combination with LDPC requires $9.5 \mathrm{~dB}$ more transmitted power to reach $90 \%$ of the corresponding peak throughput. Fig. 2 also shows that in the SNR regime of up to $35 \mathrm{~dB}$, Antipodal is the only detection and decoding scheme that can fully exploit the 256QAM modulation and reach the corresponding maximum rate.

In addition, at $33.5 \mathrm{~dB}$ our Antipodal approach achieves $56 \%$ higher throughput than "soft"-MMSE and 76\% higher throughput than "soft"-LAS approaches.

Fig. 3 compares the detection complexity of the Antipodal and the LAS detector when they are both normalized to the complexity of the MMSE detection (without including the calculation of the LLR values for MMSE). At each evaluated SNR point the plot depicts the complexity associated with the QAM modulation that maximizes the provided throughput. It is shown that in the evaluated SNR regimes the average processing requirements of the Antipodal detector are at most $4 \times$ higher than MMSE's requirements. However, the Antipodal detector, in contrast to MMSE, does not require any additional complexity to calculate soft-information (e.g., LLR values). Fig. 3 also shows that the Antipodal detector 


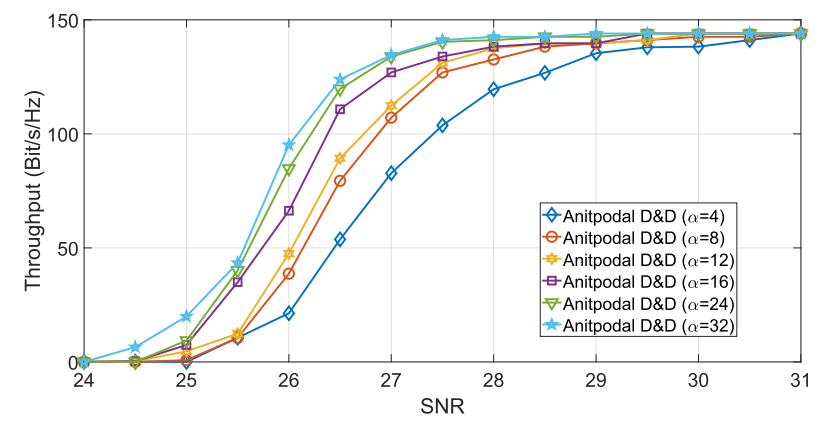

Fig. 4. Throughput of Antipodal detection and decoding with different delay constrains in a $32 \times 32$ MIMO system using 64-QAM and code rate of 0.75 .

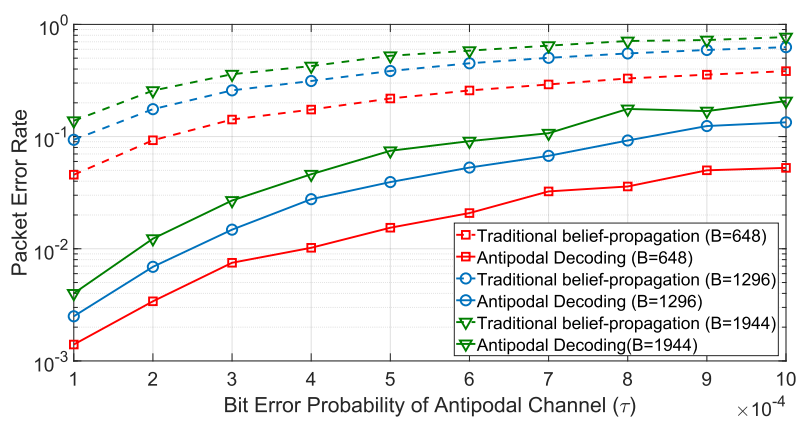

Fig. 5. Impact of bit error probability $\tau$ on the decoding performance of the Antipodal decoder (solid) and traditional belief-propagation (dashed) in Antipodal channel with 0.1 erasure probability and code rate 0.75

is consistently less complex than the non-linear LAS detector while it provides substantially higher throughput (see Fig. 2).

Fig. 4 shows the achievable throughput of Antipodal method under varying latency constraints (i.e., under distinct $\alpha$ values) for 64-QAM modulated symbols. As expected, the achievable throughput increases when allowing larger $\alpha$ values and therefore longer processing time. However, increasing the $\alpha$ value from 4 to 16 results in an SNR gain of only $1.5 \mathrm{~dB}$.

Fig. 5 shows the detection performance of the Antipodal decoder in comparison with the traditional belief-propagation decoder, for several Antipodal bit sequences. To simulate the those sequences we have used a modified Binary Ensure Channel (BEC) with erasure probability $10 \%$, and we have randomly flipped non erased bits with a probability of $\tau$. As expected, the PER of both decoders increases when increasing the error probability $\tau$. However, the Antipodal decoder outperforms traditional belief-propagation in terms of achieved PER by an order of magnitude for all tested packet lengths and over the whole evaluated range of $\tau$.

\section{CONCLUSION}

In this paper we propose the novel concept of Antipodal detection and decoding that enables the joint detection of large numbers of LDPC encoded mutually interfering information streams with practical complexity and latency requirements. We have shown that our proposed approach can substantially perform prior approaches in terms of achievable throughput. Future work, will focus on generalizing the
Antipodal detection and decoding framework to apply to several non-orthogonal transmission schemes, including the Non-Orthogonal Multiple Access ones.

\section{ACKNOWLEDGMENT}

This work has been supported by AutoAir, a UK government (DCMS) funded 5G testbed and trials project. The Authors would like to thank the members of University of Surrey 5GIC (http://www.surrey.ac.uk/5GIC) for their support.

\section{REFERENCES}

[1] J. G. Andrews and et al., "What will 5G be?" IEEE J. Sel. Areas Commun., vol. 32, no. 6, pp. 1065-1082, 2014.

[2] 3GPP, "Study on New Radio (NR) Acceess Technology; Physical Layer Aspects," 3rd Generation Partnership Project (3GPP), Technical Report (TR) 38.801, 03 2017, version 2.0.0.

[3] "Introduction to 802.11ax High-Efficiency Wireless," National Instruments, Tech. Rep., 062017.

[4] E. Viterbo and J. Boutros, "A universal lattice code decoder for fading channels," IEEE Trans. Inf. Theory, vol. 45, no. 5, pp. 1639-1642, 1999.

[5] I.-W. Lai and et al., "Searching in the delta lattice: an efficient mimo detection for iterative receivers," in Proc. IEEE Global Telecommunications Conference (GLOBECOM), 2009, pp. 1-6.

[6] B. Hassibi and H. Vikalo, "On the sphere-decoding algorithm i. expected complexity," Signal Processing, IEEE Transactions on, vol. 53, no. 8, pp. 2806-2818, 2005.

[7] J. Jaldén and B. Otterste, "On the complexity of sphere decoding in digital communications," Signal Processing, IEEE Transactions on, vol. 53, no. 4, pp. 1474-1484, 2005.

[8] K. Nikitopoulos, J. Zhou, B. Congdon, and K. Jamieson, "Geosphere: Consistently turning MIMO capacity into throughput," in Proc. ACM SIGCOMM, vol. 44, no. 4, 2014, pp. 631-642.

[9] G. Georgis, K. Nikitopoulos, and K. Jamieson, "Geosphere: An exact depth-first sphere decoder architecture scalable to very dense constellations," IEEE Access, vol. 5, pp. 4233-4249, 2017.

[10] J. Boutros, N. Gresset, L. Brunel, and M. Fossorier, "Soft-input softoutput lattice sphere decoder for linear channels," in Proc. IEEE Global Telecommunications Conference (GLOBECOM), 2003, pp. 1583-1587.

[11] K. Nikitopoulos, A. Karachalios, and D. Reisis, "Exact max-log map soft-output sphere decoding via approximate schnorr-euchner enumeration," IEEE Trans. Veh. Technol., vol. 64, no. 6, pp. 2749-2753, 2015

[12] K. V. Vardhan, S. K. Mohammed, A. Chockalingam, and B. S. Rajan, "A low-complexity detector for large mimo systems and multicarrier cdma systems," IEEE J. Sel. Areas Commun., vol. 26, no. 3, pp. 473-485, 2008.

[13] N. Srinidhi, S. K. Mohammed, A. Chockalingam, and B. S. Rajan, "Near-ML signal detection in large-dimension linear vector channels using reactive TABU search," arXiv preprint arXiv:0911.4640, 2009.

[14] S. K. Mohammed, A. Chockalingam, and B. Sundar Rajan, "A lowcomplexity near-ml performance achieving algorithm for large mimo detection," in Proc. IEEE Int. Symp. Inform. Theory (ISIT), 2008, pp. 2012-2016.

[15] R. Gowaikar and B. Hassibi, "Statistical pruning for near-maximum likelihood decoding," IEEE Trans. Signal Process., vol. 55, no. 6, pp. 2661-2675, 2007.

[16] C.-P. Schnorr and M. Euchner, "Lattice basis reduction: improved practical algorithms and solving subset sum problems," Mathematical programming, vol. 66, no. 1-3, pp. 181-199, 1994.

[17] "IEEE draft standard for information technology - telecommunications and information exchange between systems - local and metropolitan area networks - specific requirements - part 11: Wireless lan medium access control (MAC) and physical layer (PHY) specifications," pp. 1-2198, Feb 2011.

[18] D. Haccoun and G. Begin, "High-rate punctured convolutional codes for viterbi and sequential decoding," IEEE Trans. Commun., vol. 37, no. 11, pp. 1113-1125, Nov 1989. 